\title{
Die dramaturgische Funktion der Lieder in Brechts Mutter Courage und ihre Kinder
}

\section{AUGUST OBERMAYER}

Die auffallende Tendenz Brechts, dem Lied in seinem dramatischen Schaffen einen besonderen Platz einzuräumen, ist heute bereits allgemein bekannt und wird vielfach sogar als ein Charakteristikum der Brechtschen Stücke gesehen. $\mathrm{Da}$ Brecht dabei auf eine lange und nicht nur im Wiener Volkstheater wohl etablierte Tradition zurückgreifen kann und damit für die Geschichte des deutschsprachigen Dramas keine Innovation leistete, braucht trotz gelegentlicher Unsicherheiten 1 kaum noch erörtert $\mathrm{zu}$ werden. Das bewußt angewendete dramaturgische Prinzip jedoch, durch die Lieder die Handlung zu verfremden und den Gestus des Zeigens zu betonen, war in seiner Bewußtheit und Radikalität neu und ist von den Kritikern auch immer so gewertet worden.

Walter Benjamin weist bereits in der ersten Fassung seiner Studie Was ist episches Theater? (geschrieben 1931/32) auf diese wichtige Funktion der Lieder für das Brechtsche Theater hin:

Gesten erhalten wir um so mehr, je häufiger wir einen Handelnden unterbrechen. Für das epische Theater steht daher die Unterbrechung der Handlung im Vordergrunde. In ihr besteht die formale Leistung der Brechtschen Songs mit ihren rüden, herzzerreißenden Refrains. Ohne der schwierigen Untersuchung über die Funktion des Textes im epischen Theater vorzugreifen, kann festgestellt werden, daß seine Hauptfunktion in gewissen Fällen darin besteht, die Handlung - weit entfernt, sie $\mathrm{zu}$ illustrieren oder zu fördern, - zu unterbrechen. Und zwar nicht nur die Handlung eines Partners, sondern genauso die eigene. ${ }^{2}$

Die Unterbrechung der Handlung durch den 'Song', der außerhalb der Handlung stehe, trage wesentlich dazu bei, nicht so sehr Handlung, als vielmehr Zustände darzustellen. Diese Einsicht, die Benjamin 1932 aufgrund des bis dahin vorliegenden Materials möglich war, wird $1959 \mathrm{im}$ wesentlichen von Reinhold Grimm bestätigt:

Sie (die Songs, Anm. Verf.) sind eines der Hauptmittel der Verfremdung. Ihre Wirkung beruht auf dem Wechsel der Form, wie er auch für den Übergang von der Prosa zum Vers gilt. ${ }^{3}$ 
Im Brechtschen Werk läßt sich aber eine Entwicklung in der Vẹrwendung der Lieder feststellen. Waren die Lieder vorerst Einlagen, die außerhalb der Handlung ihre Funktion als Mittel der Verfremdung erfüllten - das war so als Benjamin seinen Aufsatz schrieb - werden sie später allmählich in die Handlung integriert. Sie sind nunmehr unmittelbar mit der Handlung verbunden, wachsen vielfach aus ihr heraus. Hans Mayer hat das bereits 1952 überzeugend an der Verwendung des 'Salomonsong' in der Dreigroschenoper und in Mutter Courage demonstriert. 4

Somit kommt den Liedern aber eine zusätzliche Funktion zu. Neben ihrer Leistung als Mittel der Verfremdung übernehmen sie auch eine strukturierende Aufgabe: sie sind Teil des Handlungsgefüges, strukturieren das Stück von 'innen', auf der Ebene der Handlung. Das heißt, eine von außen aufgesetzte Gliederung wird allmählich zu einer organischen, völlig ins Stück integrierten Struktur.

Brecht selbst hat jedoch die Meinung vertreten, daß es in Mutter Courage Lieder gebe, die nicht aus der Handlung herauswachsen und um da keinen Zweifel aufkommen zu lassen, hat er bei seinen eigenen Inszenierungen ein Musikemblem verwendet:

Um zum Musikalischen umzuschalten, der Musik das Wort zu erteilen, ließen wir jedesmal, wenn ein Lied kam, das nicht unmittelbar aus der Handlung herauskam oder, aus ihr herausgekommen, deutlich außen blieb, vom Schnürboden ein Musikemblem herunter, bestehend aus Trompete, Trommel, Fahnentuch und Lampenbällen, welche aufleuchteten. $\cdots$

es diente uns dazu, den Wechsel zu einer andern ästhetischen Ebene, der musikalischen, sichtbar zu machen, so daß nicht der falsche Eindruck entstand, die Lieder 'wüchsen aus der Handlung heraus', sondern der richtige Eindruck, sie seien Einlagen. 5

Somit ergibt sich die Frage, welche Lieder aus der Handlung herauswachsen und welche als Einlagen zu verstehen sind. Es drängt sich auch der Verdacht auf, daß Brecht alle Lieder als Einlagen verstanden wissen wollte, da sie doch alle auf der 'anderen ästhetischen Ebene, der musikalischen' angesiedelt sind. Aus den Anmerkungen geht mit Sicherheit hervor, daß das Musikemblem für den 'Salomonsong' in der neunten Szene verwendet wurde. 6

Neben vielen Einzelbeobachtungen, auf die im Laufe der Untersuchung noch hinzuweisen sein wird, gibt es zwei Studien, die sich eingehend mit dem Lied im Werke Brechts beschäftigt haben. ${ }^{7}$

Stupinski hält am Begriff der 'Einlage' fest, sieht jedoch einen Wandel in der inhaltlichen Funktion der Lieder von der im Lied zum Ausdruck gebrachten Revolte als 'Bürgerschreck' hin zum 'reflexiv aufgebaute(n) Lied, das dem Streben zur Tiefe, dem Lösen von Rätseln und der Suche nach neuen Wegen dient', 8 während Thole den Wandel von der 'Einlage' zum 'funktionalen Be- 
standteil der Fabel' erkennt, bei seiner Systematisierung der verschiedenen Funktionen der Lieder jedoch vielfach an der Oberfläche bleibt.

In Mutter Courage und ihre Kinder wird zwölfmal gesungen, allerdings sind die Lieder nicht so verteilt, daß jeder der zwölf Szenen ein Lied zukommt. Die Szenen fünf und elf sind liedlos, während denSzenen drei und zwölf je $z$ wei Lieder zugeteilt sind. Dieser häufige Einsatz von Liedern hat verschiedentlich zu Kritik geführt, ${ }^{9}$ ist aber auch positiv interpretiert worden. Theodor Luthard sieht den 'Song' als Schlüssel zur dramatischen Grundkonzeption des Stückes, 10 Walter Schäfer versuchte eine Interpretation, die von den Liedern ausgeht und so allmählich ins Stück eindringt. ${ }^{11}$

Trotz der Vielzahl von wissenschaftlichen Äußerungen zu den Liedern in Mutter Courage, wobei eine Konzentration auf das 'Lied der Mutter Courage', das 'Lied von der großen Kapitulation' und den 'Salomonsong' festzustellen ist, ist die Leistung der Lieder für die dramatische Struktur des Stückes bisher nicht untersucht worden.

Seit der aufschlußreichen Arbeit von Reinhold Grimm, "Pyramide und Karussell", die das Stück Mutter Courage als eine Aneinanderreihung von 'Stückchen im Stück', die zu "einer Art Kreisform"12 zusammengeschlossen sind, ausweist, ist es Gemeinplatz der Brechtforschung geworden, von der Kreisstruktur der Mutter Courage zu sprechen.

Mutter Courage, nicht fähig aus ihrer Situation Lehren zu ziehen, bewegt sich mit ihrem Wagen nicht nur auf der Drehscheibe im Kreis, sie geht buchstäblich 'im Kreis'. So wie sie am Beginn des Stückes dem Krieg nachläuft, geschieht das das ganze Stück hindurch und am Ende des Stückes findet sie sich in genau derselben Situation. Eben diese Situation wird durch den Einsatz und die Anordnung der Strophen des 'Liedes der Mutter Courage' deutlich. Die formale Leistung dieses Liedes als Rahmen und Leitmotiv ist schon erkannt worden.

Brecht stellt einen Ausschnitt aus dem Dreißigjährigen Krieg, die Jahre 1624-1636 dar, einen Abschnitt also, der durch den Einsatz des Liedes in der ersten und zwölften Szene betont wird. Die erste Szene zeigt Mutter Courage bei ihrem Auftritt mit diesem Lied in den Krieg ziehen, sie läuft in der siebten Szene, auf dem Höhepunkt ihrer geschäftlichen Laufbahn mit ebendiesem Lied dem Krieg hinterher, sie benutzt es am Ende der achten Szene, diesmal gehen die Geschäfte etwas schlechter, abermals, um dem Krieg nachzulaufen, sodaß es am Ende des Stückes nicht überrascht, wenn das Lied wieder erklingt, diesmal vom Regiment gesungen und Mutter Courage, am absoluten Tiefpunkt ihrer Karriere, genau das tut, was sie immer gemacht hat: sie läuft abermals hinter dem Krieg her. Diesem formalen Schlußpunkt des Stückes wurde von Brecht 1949 in der Berliner Aufführung ein korrespondierender Beginn beigegeben, indem er das 'Lied der Mutter Courage' in einer Art Vorspiel gleich zu Beginn des Stückes singen ließ,13 was zwar formal einen perfekten Rahmen ergab, die Entwicklung des Liedes aus dem Dialog aber verhinderte. Im gedruckten Text ${ }^{14}$ 
folgt das Lied unmittelbar auf die knappe Antwort der Courage, die diese auf die Frage des Feldwebels: "Wer seid ihr?" (S. 1350) erteilt. Es ist seinem Charakter nach Auftrittslied im Sinne der Tradition der Barockoper, seiner Aussage nach Rollenlied, wie es die Wiener Volkskomödie kennt. Brecht selbst benützt die Termini 'Auftrittslied' und 'Geschäftslied':

Wir hatten das Lied vorgesehen als theatralisches Auftrittslied, schwungvoll und frech - wir dachten an die letzte Szene des Stücks. Aber die Weigel wünschte es als Geschäftslied, Lied der aktualen Repräsentation, und sie schlug vor, es zur Darstellung des langen Wegs in den Krieg zu benutzen. 15

Wie immer, das Lied, wie es im Text gedruckt erscheint, zeigt Merkmale beider Liedtypen: es ist Auftrittslied, in dem sich die Bühnenfigur dem Publikum präsentiert und auch Rollenlied, da der Zuschauer über die Art der Geschäfte und über die Einstellung der Courage zu diesen Geschäften unterrichtet wird. Es zeigt Mutter Courage als kluge Geschäftsfrau, die weiß, wie sie sich sprachlich zu verhalten hat. Sie wendet sich an die 'Hauptleut', schmeichelt ihnen und weiß, daß sie so ins Geschäft kommen wird: das Lied wird also zu einem Werbesong:

Ihr Hauptleut, laßt die Trommel ruhen

Und laßt eur Fußvolk halten an (S. 1350)

"Das ist der Geschäftstrick der Marketenderin, sie weiß.. .daß die Niederen die Sprache der Oberen sprechen müssen". 16

Das Lied, wie es im Text gedruckt wurde, wächst aus dem Dialog heraus. Die Frage des Feldwebels wird mit dem lapidaren 'Geschäftsleut' beantwortet, aber erst das Lied gewährt Einblick, um welche Art Geschäfte es sich da handelt. Es sind Geschäfte mit dem Krieg und durch den Krieg. Sie präpariert den Hauptleuten das Fußvolk für den Tod und den Marsch in den 'Höllenschlund' - gegen Entgelt, versteht sich.

Ihr Hauptleut, eure Leut marschieren

Euch ohne Wurst nicht in den Tod.

Laßt die Courage sie erst kurieren

Mit Wein von Leibs- und Geistesnot.

Kanonen auf die leeren Mägen

Ihr Hauptleut, das ist nicht gesund.

Doch sind sie satt, habt meinen Segen

Und führt sie in den Höllenschlund. (S. 1351)

In dem insgesamt dreimal wiederkehrenden Refrain:

Das Frühjahr kommt. Wach auf, du Christ!

Der Schnee schmilzt weg. Die Toten ruhn. (S. 1351)

vollzieht sich die Poetisierung des Gewerbes der Courage durch die "lyrische 
Heroisierung des Berufes jener kleinen Hyäne des Krieges". 17 Diese Poetisierung wird aber nicht konsequent durchgehalten. Die folgenden beiden Verse sinken sofort wieder auf das Niveau der Mächtigen, die das 'Höhere' in Moderation goutieren mögen, zuviel davon aber keinesfalls akzeptieren würden:

Und was noch nicht gestorben ist

Das macht sich auf die Socken nun. (S. 1351)

In der Rückkehr zur colloquialen Ebene vollzieht sich die Entzauberung. Das kann durchaus als Verkaufsjargon gewertet werden, der sich der Mentalität der Kundschaft anpaßt.

In der ersten Strophe des Liedes vollzieht sich der Wechsel vom eigentlichen Text der Strophe zum Kehrreim sprunghaft. Das Fußvolk muß Schuhe haben, 'in denens besser laufen kann' - in welche Richtung wird ironischerweise nicht gesagt - Mutter Courage kann diese Schuhe bereitstellen. Der Kehrreim spottet der Wirklichkeit, kann aber immerhin noch als die notwendige Mischung von 'Dreistigkeit und Pathos' aufgefaßt werden, deren sich die kleinen Leute bedienen müssen, um zu überleben.

In der zweiten Strophe wird klar, daß der Kehrreim bitterer Hohn ist:

Doch sind sie satt, habt meinen Segen

Und führt sie in den Höllenschlund.

Das Frühjahr kommt. Wach auf du Christ! (S. 1351)

"In den Sprüngen zwischen den Strophen und dem Kehrreim tut sich die wahre Haltung der 'Mutter' Courage kund: ihre Menschenverachtung". 18 Es wird einsichtig, daß Mutter Courage den merkantilen Charakter des Krieges erkannt hat und ganz bewußt durch die poetische Verbrämung unmoralischer Aktionen ihrem Profitstreben eine gewisse Respektabilität verleihen möchte.

In der dritten Strophe des Liedes, die das Stück beschließt und nun nicht mehr von Mutter Courage, sondern vom Regiment gesungen wird, ist bereits deutlich ein Denkschritt vollzogen worden. Der Übergang zum Kehrreim vollzieht sich nunmehr auch fugenlos. Der 'g'meine Mann' hat erkannt, daß für ihn im Krieg kein Profit zu erhoffen ist, das Wunder, das im Kehrreim evoziert wird, ist im Strophentext bereits deutlich angesprochen:

Mit seinem Glück, seiner Gefahre

Der Krieg, er zieht sich etwas hin.

Der Krieg, er dauert hundert Jahre

Der g'meine Mann hat kein Gewinn.

Ein Dreck sein Fraß, sein Rock ein Plunder!

Sein halben Sold stiehlts Regiment.

Jedoch vielleicht geschehn noch Wunder:

Der Feldzug ist noch nicht zu End! 
Das Frühjahr kommt! Wach auf, du Christ!

Der Schnee schmilzt weg! Die Toten ruhn!

\section{...

Es ist allerdings möglich, wie Wölfel vorgeschlagen hat, die Zeile 'Der Feldzug ist noch nicht zu End!', vor allem wegen des Rufzeichens am Ende, als revolutionäre Drohung zu verstehen. In diesem Sinne wäre der Beginn des Kehrreims: "Das Frühjahr kommt! Wach auf du Christ! Der Schnee schmilzt weg! Die Toten ruhn!" - auch hier hat Brecht jetzt überall Rufzeichen gesetzt - als Aufruf zur revolutionären Tat, für die das Frühjahr die rechte Zeit zu sein scheint, zu lesen. Natürlich versteht Mutter Courage das Lied nicht so. Für sie ist es das Motiv zum Aufbruch in den Krieg. Für das Publikum hat sich aber deutlich sichtbar eine Entwicklung vollzogen, die Situation ist nicht dieselbe wie am Beginn des Stückes. Ein Erkenntnisprozeß hat stattgefunden und wird im Lied ausgedrückt. Der sich damit auftuende Hoffnungsschimmer ist Zuversicht für das Publikum, daß Erkenntnis sozialer Zusammenhänge auch dem kleinen Mann möglich sein sollte. Die im Lied zum Ausdruck gebrachte Progression wird aber von der Courage nicht nur nicht mitvollzogen. Im Gegenteil, sollte ihre zynische Demaskierung des Krieges zur Veränderung auffordern, was am Ende des Stückes zumindest auf der Ebene der Erkenntnis erreicht wurde, wenngleich die dramatis personae auch keine Konsequenzen daraus ziehen, so handelt die Courage dieser Entwicklung ständig entgegen. Durch ihre willige Teilnahme am Krieg, ihre bereitwillige Anpassung und Hilfeleistung aus purem Geschäftsinteresse retardiert sie diesen Vorgang geradezu. Sie trägt durch ihr Verhalten entschieden da$\mathrm{zu}$ bei, den status quo zu perpetuieren und zu zementieren. Die Aktionen der Mutter Courage sind den im Lied ausgedrückten Intentionen gegenläufig.

In der zweiten Szene singt der kühne Sohn Eilif im Vollgefühl seines Erfolgs, voll Übermut und überschäumender Ausgelassenheit das 'Lied vom Weib und dem Soldaten' und führt dazu einen verwegenen Kriegstanz auf. Auch dieses Lied erwächst aus dem Dialog: "Da kann ich ein Lied" (...) "Es heißt: Das Lied vom Weib und dem Soldaten" (S. 1366) und dient auch als akustisches Erkennungszeichen zwischen Mutter und Sohn. Eilif singt die beiden Strophen, in denen der übermütige Soldat die Warnung des Weibes lachend in den Wind schlägt:

Das Schießgewehr schießt, und das Spießmesser spießt

Und das Wasser frißt auf, die drin waten.

Was könnt ihr gegen Eis? Bleib weg, 's ist nicht weis!

Sagte das Weib zum Soldaten.

Doch der Soldat mit der Kugel im Lauf

Hörte die Trommel und lachte darauf:

Marschieren kann nimmermehr schaden!

Hinab nach dem Süden, nach dem Norden hinauf

Und das Messer fängt er mit Händen auf! 
Sagten zum Weib die Soldaten. (S. 1366)

Mutter Courage antwortet mit dem Rest des Liedes. Die Warnung des 'Weibes' hat sich als gerechtfertigt erwiesen, der Soldat ist tot und hat dem Weib nichts mehr zu sagen:

Er verging wie der Rauch, und die Wärme ging auch

Und es wärmten sie nicht seine Taten.

Ach, bitter bereut, wer des Weisen Rat scheut!

Sagte das Weib den Soldaten. (S. 1367)

Das Lied ist ein negativer Kommentar zu Eilifs Verhalten. Der kühne Soldat Eilif, der die Warnung des 'Weibes' (der Mutter) lachend abtut, wird gerade an dieser Kühnheit zugrunde gehen, sinnlos 'wie der Rauch' vergehen.

Liedintention und Handlungsrichtung sind abermals gegenläufig.

In der dritten Szene singt Yvette, die Lagerhure, die professionell Liebende, das 'Lied vom Fraternisieren' als Warnung vor der Liebe. Kattrin soll der Liebe gegenüber 'abgehärtet' werden. Der Zusammenhang mit der dramatischen Handlung ist damit hergestellt, daß der im Lied vorkommende Koch mit dem im Stück vorkommenden Koch identisch ist und auch damit, daß Mutter Courage eine Zeile des Liedes dann in ihren Dialog übernimmt: "Die Liebe ist eine Himmelsmacht, ich warn dich". (S. 1372)

Yvette wurde von einem feindlichen Soldaten verführt, verlassen und so in die Prostitution getrieben:

Die Liebe, die ich spürte

War eine Himmelsmacht.

Meine Leut habens nicht begriffen

$\mathrm{DaB}$ ich ihn lieb und nicht veracht.

In einer trüben Früh

Begann mein Qual und Müh

Das Regiment stand im Geviert

Dann wurd getrommelt, wies der Brauch

Dann ist der Feind, mein Liebster auch

Aus unsrer Stadt marschiert. (S. 1371)

Yvette, die sich hier über ihr Schicksal beklagt, wird dann die einzige sein, die vom Krieg tatsächlich profitiert.

Die Liedintention wird aber abermals durch direkte Aktion der Handlung aufgehoben. Kattrin ist in keiner Weise gewarnt. Nachdem sie vom Feldprediger als "einnehmende Person" (S. 1373) apostrophiert wurde, geht sie "von der Wäsche weg, auf den Hut zu. Sie hebt ihn auf und setzt sich, die roten Schuhe anziehend"' (S. 1373 f) Sie beginnt, Yvettes Hut auf dem Kopf, in den roten Schuhen, den Symbolen der Prostitution, herumzustolzieren und weigert sich, diese an Yvette abzuliefern:

Yvette: Und wo sind die roten Schuh? (Sie findet sie nicht, weil Kattrin 
die Füße unter den Rock zieht). . (S. 1377)

Das in derselben Szene vom Feldprediger vorgetragene 'Horenlied' wird von vornherein als Vergleich angelegt. Schweizerkas ist eben von den Häschern abgeführt worden und der Feldprediger vergleicht diese Situation mit der Passion Christi. Das Lied erwächst direkt aus dem Dialog, wirkt historisierend, sodaß das Leiden einen überindividuellen göttlichen Sinn erhält:

Solche Fäll, wos einen erwischt, sind in der Religionsgeschicht nicht unbekannt. Ich erinner an die Passion von unserm Herrn und Heiland.

Da gibts ein altes Lied darüber. (S. 1384)

Die Wiedergabe der Passion, gipfelnd in den Versen:

Solches stellen sie uns an

Mit dem Menschensohne. (S. 1385)

hat schon im Tempuswechsel vom durchgehenden Imperfekt zum Präsens 'stellen' einen direkten Bezug zum Schicksal des Schweizerkas und ist in seiner Intention auf Trost, Erbauung und Resignation gerichtet. "Das Horenlied feiert in Christus das Leiden als Gottesgeschick, so daß dem Leiden in der Welt selbst göttliche Heiligkeit zugesprochen werden kann". 19

$\mathrm{Da}$ vom Tode Christi die Rede ist, ist dem Zuschauer der Tod Schweizerkas fast schon zur Gewißheit geworden. Mutter Courage tröstet sich aber in keiner Weise dämit, daß andere Söhne, selbst der Sohn Gottes, ein ähnliches Schicksal erlitten haben. Sie ist weder gefaßt noch resigniert. Der auf Trost, Erbauung und Besinnung gerichteten Liedintention wird sofort durch fieberhafte Aktivität der Mutter Courage entgegengewirkt:

Mutter Courage kommt aufgeregt: Es ist auf Leben und Tod. . . (S. 1385) Der Tod Eilifs ist auch nicht "das Ergebnis einer höheren, in Jesu Leiden sanktionierten Notwendigkeit, sondern die Folge der Geschäftstüchtigkeit seiner Mutter".20

Die handlungsarme vierte Szene ist dominiert vom 'Lied von der großen Kapitulation'. Die Courage und ein junger Soldat wollen sich beschweren. Während sie beide warten singt sie ihm ein Lied: "Ich werd Ihnen was erzählen von der Großen Kapitulation" (S. 1394) Dabei überzeugt sie den Soldaten und sich selbst von der Sinnlosigkeit eines solchen an sich berechtigten Unternehmens. Lehrend lernt sie sich 'nach der Decke zu strecken'.

Ähnlich wie im 'Lied der Mutter Courage' wird der Abstand zwischen Strophentext und Kehrreim von Strophe zu Strophe verringert. Findet man in der ersten Strophe noch einen deutlichen Kontrast zwischen dem Anspruch:

Einst, im Lenze meiner jungen Jahre

Dacht auch ich, daß ich was ganz Besondres bin.

(Nicht wie jede beliebige Häuslerstochter, mit meinem Aussehn und Talent und meinem Drang nach Höherem!) 
Und bestellte meine Suppe ohne Haare

Und von mir, sie hatten kein Gewinn.

(Alles oder nix, jeden falls nicht den Nächstbesten, jeder ist

seines Glückes Schmied, ich laß mir keine Vorschriften

machen!)

und dem Kehrreim:

Doch vom Dach ein Star

Pfiff: wart paar Jahr!

Und du marschierst in der Kapell

Im Gleichschritt, langsam oder schnell

Und bläsest deinen kleinen Ton:

Jetzt kommt er schon.

Und jetzt: das Ganze schwenkt!

Der Mensch denkt: Gott lenkt -

Keine Red davon! (S. 1394f)

so vereinigt sich der Text der letzten Strophe fugenlos mit dem Kehrreim:

Viele sah ich schon den Himmel stürmen

Und kein Stern war ihnen groß und weit genug.

(Der Tüchtige schafft es, wo ein Wille ist, ist ein Weg, wir werden den Laden schon schmeißen).

Doch sie fühlten bald beim Berg-auf-Berge-Türmen

Wie doch schwer man schon an einem Strohhut trug.

(Man muß sich nach der Decke strecken!)

(S. $1395 \mathrm{f}$ )

Der Soldat und Mutter Courage, die beide mit der festen Absicht sich zu beschweren gekommen waren und sich schon vor dem Offizierszelt eingefunden haben, werden in dieser Absicht vom Lied gestört. Sie beschweren sich nicht. Der von der Courage 'angepaßte' Soldat wird in der elften Szene als Fähnrich ihre Tochter erschießen lassen. "Die Bekehrung der Courage hat nicht den Charakter mütterlicher Fürsorge gegenüber einem noch unerfahrenen jungen Menschen, sondern den der Verführung".21

In der sechsten Szene steht der Krieg für einen Augenblick still. Tilly, der kaiserliche Feldherr, ist gefallen und wird mit Pomp zu Grabe getragen. Der Krieg 'verschnauft sich', stagniert für eine Weile, doch wird gerade in dieser Situation das hastig vorwärtsdrängende Reiterlied gesungen:

Ein Schnaps, Wirt, schnell, sei g'scheit!

Ein Reiter hat kein Zeit.

$\mathrm{Mu} ß$ für sein Kaiser streiten. (S. 1402)

Die dreimal wiederkehrende Zeile 'Ein Reiter hat kein Zeit' wirkt der allgemeinen Situation und auch dem reflexiven Verhalten der Courage, die vor der wichtigen Entscheidung steht, ob sie Vorräte einkaufen soll oder nicht, kontrastiv 
entgegen. Unmittelbar vor dem Lied ist von der langen Dauer des Krieges die Rede. Der szenische Zusammenhang ergibt: "das lange Leben des Krieges bedingt das kurze Leben des Kriegers". 22

Dein Spruch, Pfaff, schnell, sei g'scheit!

Ein Reiter hat kein Zeit.

Er muß fürn Kaiser sterben. (S. 1402)

Das 'Lied vom Salomon' der neunten Szene wird als Bettellied vom Koch vorgetragen. Er erhofft sich damit eine 'warme Supp' zu sichern und ist auch erfolgreich. Damit ist der szenische Zusammenhang gegeben. Darüber hinaus warnt das Lied vor den verderblichen Auswirkungen großer Tugenden: "Alle Tugenden sind nämlich gefährlich auf dieser Welt, wie das schöne Lied beweist, man hat sie besser nicht und hat ein angenehmes Leben und Frühstück, sagen wir, eine warme Supp" (S. 1425). Die Weisheit, die Kühnheit, die Redlichkeit, die Heiligkeit und die Frömmigkeit sind, wie an Beispielen aus der Geschichte demonstriert wird, ein regelrechter Luxus, den man sich nicht leisten kann, will man den Krieg überleben. Die stets wiederkehrende Zeile 'Beneidenswert, wer frei davon!' weist eindringlich darauf hin. Im Verlaufe des Stückes bewahrheitet sich diese These auch: Eilif stirbt an exzessiver Kühnheit, Schweizerkas an übertriebener Redlichkeit und Kattrin an übergroßem Mitleid.

In der neunten Szene aber läuft der Song der dramatischen Handlung direkt entgegen. Am Ende der Szene entscheidet sich Mutter Courage gegen die sichere Bleibe und das gesicherte Einkommen, gegen die Wirtschaft in Utrecht und gegen den Koch. Sie entscheidet sich für ihre Tochter Kattrin und die damit verbundene unsichere Lebensweise. Sie setzt also gerade dann, wenn das Lied eindringlich vor den Gefahren, die mit den Tugenden verbunden sind, warnt, eine tugendhafte Handlung größten Umfanges. ${ }^{23}$

Die zehnte Szene besteht bloß aus einem Lied. Eine Stimme malt das idyllische Bild der ergötzlichen Rose im Garten, der wohlbestellten sicheren Bleibe, des sturmgesicherten Hauses im Winter.

Uns hat ein Ros ergetzet

Im Garten mittenan

...

Und wenn die Schneewind wehen

Und blasen durch den Tann

Es kann uns wenig g'schehen

Wir habens Dach gerichtet

Mit Moos und Stroh verdichtet. (S. 1429)

Der egozentrisch zufriedene, beinahe verklärte Grundgestus des Liedes "Wohl denen, die ein Garten han", "Wohl denen, die ein Dach jetzt han" steht in deutlichem Kontrast zur ruinösen Ungeborgenheit der Courage und ihrer Tochter. Beide ziehen den 'immer zerlumpteren Heeren' nach, ohne 
Bleibe, ohne Schutz, ohne Sicherheit. "Mutter Courage und Kattrin haben eingehalten, um zuzuhören, und ziehen dann weiter". (S. 1429) Die deutliche Kenntnisnahme des Liedes durch die agierenden Personen stellt den szenischen Zusammenhang her. Es ist auch "lyrisches Resumee der tugendhaften Handlung der Courage, die gerade zuvor ein Heim ausgeschlagen hat, um ihre Tochter nicht verlieren zu müssen". 24

In der letzten Szene endlich singt Mutter Courage das Wiegenlied 'Eia popeia Was raschelt im Stroh' (S. 1436). Kattrin ist tot, doch die Courage weigert sich, dies wahrzuhaben: "Vielleicht schlaft sie" (S. 1436) "Der Gedanke, der dem Lied zugrundeliegt, ist ein mörderischer: Das Kind dieser Mutter sollte es besser haben als andere Kinder anderer Mütter". 25

Nachbars Bälg greinen

Und meine sind froh.

Nachbars gehn in Lumpen

Und du gehst in Seid

Ausn Rock von einem Engel

Umgearbeit'. (S. 1436)

Der lebenden Tochter konnte sie nicht bieten, was sie jetzt der toten anbietet, sie konnte ihr nicht einmal das Leben erhalten. "Dem Kind, dem das Gewöhnlichste versagt blieb, wurde das Außergewöhnliche versprochen"26 - aber es ist zu spät. Ein letztes Mal versucht die Courage als 'gute' Mutter zu agieren, während sie durch ihre geschäftlichen Unternehmungen zur Mörderin ihres Kindes geworden ist.

Somit kann zweierlei festgestellt werden:

(a) textliche Evidenz zeigt, daß die Lieder szenisch integriert sind, aus dem Handlungszusammenhang und dem Dialog herauswachsen, also keine 'Einlagen' sind, nicht außerhalb der Handlung bleiben.

(b) die Liedintention und die dramtische Handlung verhalten sich zueinander gegenläufig.

Damit kann der Auffassung, die Lieder in Brechts Stücken seien "lediglich geschaffen, um die Handlung willkürlich zu zerreißen, um beim Zuschauer die Illusion zu zerstören" 27 zumindest für den Fall der Mutter Courage widersprochen werden.

Volker Klotz führt für das atektonische Drama den Begriff des 'kreisenden Songs' ein, worunter er Lieder versteht, die sich karussellartig um immerwiederkehrende Verse oder Refrains drehen. Er beobachtet das unter anderem auch bei Mutter Courage und folgert, daß solche Lieder "menschliche Haltungen und Eigenschaften exemplarisch zeigen".28

Diese Qualität des Exemplarischen ist zweifelsohne vorhanden, aber sie ist nicht exklusiv. Sie ergibt sich aus der kontextuellen Einordnung des Liedes und seiner strukturbildenden Funktion innerhalb der Szene und des Stückes als 
Ganzem, als Folge oder Ergebnis der Placierung eines bestimmten Liedes an einem bestimmten Platz innerhalb des Stückes.

Keineswegs läßt sich daraus ableiten, wie Peter Pütz das versucht hat, die Lieder hätten "damit auch keinen festen Platz an einer bestimmten Stelle des Stücks, sondern können verschoben und wiederholt werden". 29

Für Mutter Courage zumindest hat sich diese Annahme als gänzlich unhaltbar erwiesen. Die Lieder haben einen bestimmten und fixierten Platz innerhalb der Szene und damit innerhalb des Stückes. Durch die retardierende Tendenz des Liedes im Kontrast zum Motorischen der Handlung wird Spannung erzeugt. So wie innerhalb der einzelnen Szenen kleine Spannungsbögen angelegt sind, wird durch das Rahmenlied ein großer Spannungsbogen um das ganze Stück gelegt, sodaß trotz des chronikartigen Ausschnittes ein teleologisches Fortschreiten des Stückes gegeben ist, das sich besonders durch den in der dritten Strophe des 'Liedes der Mutter Courage' vollzogenen Denkschritt manifestiert.

Die Lieder haben sich als wichtige Strukturelemente erwiesen, die dazu beitragen, daß die 'Kreisform' nicht in linearer Progression erreicht wird, sondern durch immer wieder notwendig werdende Überrundung kleiner Rückschritte. Dadurch wird die Handlung zwar unterbrochen, aber eben auf der Ebene der Handlung, nicht durch 'Einlagen', nicht durch Textstellen, die 'deutlich außen bleiben',. sondern durch Lieder, die eindeutig aus dem szenischen Zusammenhang und dem Dialog herauswachsen.

"Das Lied ist ein Bestandteil der Fabel. Zugleich ist es ein selbständiges Wesen. Außerhalb des Stückes ist es verständlich und nicht verständlich: es ist verständlich als lyrisches Gebilde, unverständlich als dramaturgisches Element. Von seiner lyrischen Seite her betrachtet, hat es andere Eigenschaften als von der dramatischen her; die Eigenschaften widersprechen sich, und es hat sie alle (...) Im Zusammenhang mit der Aufführung verliert es seine Unabhängigkeit, wächst aber über sich hinaus und profitiert vom Wert der gesamten Sache. Es folgt den.gleichen Regeln wie jeder Teil eines organisierten Ganzen, also wie die Szene eines Stücks, der Abschnitt einer Szene, der einzelne Satz. Es unterliegt, um es in ganz simple Rede zu fassen, dem Gesetz der Einheit von Kontinuität und Diskretheit. (. . .) Das richtige Theaterlied gehört, unauswechselbar, an seine bestimmte Stelle im Stück. Das richtige Theaterlied ist ein lebensfähiges poetisches Individuum".30

Das ist die Ansicht Peter Hacks über Lieder zu Stücken. Brecht, der Stückeschreiber, scheint in Mutter Courage genau dieses Konzept erfüllt zu haben, obwohl Brecht, der Regisseur und Theoretiker, alles tat, um den "falschen Eindruck", "die Lieder wüchsen aus der Handlung heraus" zu vermeiden und den "richtigen Eindruck, sie seien Einlagen"31 zu fördern. Wie im Falle Brecht schon öfters festgestellt werden konnte, hat sich auch hier der Künstler seine Unabhängigkeit gegenüber dem Theoretiker bewahrt. 
Anmerkungen

*Diese Arbeit konnte mit Unterstützung der Alexander von Humboldt-Stiftung verfaßt werden.

1 Vgl. Lore Lucas, Dialogstrukturen und ihre szenischen Elemente im deutschsprachigen Drama des 20. Jahrhunderts (-Abhandlungen. zur Kunst-, Musik- und Literaturwissenschaft 72), Bonn 1969, S. 117: "Mit dem Song hat Brecht ein neues Gestaltungsmittel eingeführt, das dramaturgisch wie szenisch als das am meisten charakteristische Element seines Verfremdungsgesprächs gelten darf'.

2 Walter Benjamin, Versuche über Brecht. Herausgegeben und mit einem Nachwort versehen von Rolf Tiedemann (-edition suhrkamp 172), Frankfurt a.M. 1966, S. 9f.

3 Reinhold Grimm, Bertolt Brecht. Die Struktur seines Werkes (-Erlanger Beiträge zur Sprach- und Kunstwissenschaft), Nürnberg 1959, S. 59.

4 Hans Mayer, "Anmerkung zu einer Szene aus Mutter Courage" in Materialien zu Brechts 'Mutter Courage und ihre Kinder' (-edition suhrkamp 50), Frankfurt a.M. 1976, S. 149-156.

5 Bertolt Brecht, "Anmerkungen zur Aufführung 1949" in Materialien, a.a.O., S. 12.

6 Materialien, a.a.O., S. 12.

7 Zbigniew Stupiński, Die Funktion des 'Songs' in den Stücken Bertolt Brechts (-Uniwersytet Im. Adama Mickiewicza W Poznaniu, Wydziat Filologiczny, Seria Filologia Germańska Nr 13), Poznań 1972.

Bernward Thole, Die 'Gesänge' in den Stücken Bertolt Brechts. Zur Geschichte und Asthetik des Liedes im Drama (Göppinger Arbeiten zur Germanistik 102), Göppingen 1973.

8 Stupiński, a.a.O., S. 71.

9 Darauf weist schon Hans Mayer, a.a.O., S. 151 hin.

10 Theodor Luthardt, "Der Song als Schlüssel zur dramatischen Grundkonzeption in Bertolt Brechts Mutter Courage und ihre Kinder", Wissenschaftliche Zeitschrift der Friedrich-Schiller-Universität Jena 7 (1957/58), Gesellschafts- und sprachwissenschaftliche Reihe H. 1, S. 119-122.

11 Walter Schäfer, "War der Weg über die Lieder ein Umweg? Bert Brecht: Mutter Courage und ihre Kinder", WW 14 (1964), S. 407-413.

12 Reinhold Grimm, "Pyramide und Karussell" in Reinhold Grimm, Strukturen. Essays zur deutschen Literatur, Göttingen 1963, S. 32.

13 Materialien, a.a.O., S. 17. 
14 Zitiert wird nach dem Text: Bertolt Brecht, Gesammelte Werke in 20 Bänden (-werkausgabe edition suhrkamp), Frankfurt a.M. 1968, Bd 4, S. 1347-1438.

15 Materialien, a.a.O., S. 18.

16 Friedrich Wölfel, "Bertolt Brecht. Das Lied der Mutter Courage" in Wege zum Gedicht II. Interpretationen von Balladen. Herausgegeben von Rupert Hirschenauer und Albrecht Weber, München 1968, S. 539.

17 Andrzej Wirth, "Die Funktion des Songs in Mutter Courage und ihre Kinder" in Materialien, a.a.O., S. 158.

18 Wölfel, a.a.O., S. 540.

19 Helmut Jendreiek, Bertolt Brecht. Drama der Veränderung, Düsseldorf 1969, S. 170.

20 Jendreiek, a.a.O., S. 202.

21 Jendreiek, a.a.O., S. 182.

22 Alfred Reisinger, Bertolt Brechts 'Mutter Courage und ihre Kinder'. Ein Beitrag zur Erkenntnis der ästhetischen Struktur des literarischen Kunstwerks, Diss. Wien 1973, S. 83.

23 Darauf hat Hans Mayer, a.a.O., schon 1952 hingewiesen.

24 Reisinger, a.a.O., S. 94.

25 Materialien, a.a.O., S. 76.

26 Materialien, a.a.O., S. 76.

27 Luthardt, a.a.O., S. 120 wendet sich schon gegen diese Auffassung.

28 Volker Klotz, Geschlossene und offene Form im Drama (-Literatur als Kunst), München 1960, S. 212.

29 Peter Pütz, Die Zeit im Drama. Zur Technik dramatischer Spannung, Göttingen $1977^{2}$, S. 143.

30 Peter Hacks, “Über Lieder zu Stücken”, Sinn und Form 14 (1962), S. 425.

31 Materialien, a.a.O., S. 12. 